Reimar Banis

\section{Einleitung}

1997 wurde die Psychosomatische Energetik (PSE) erstmals bei der Medizinischen Woche in Baden-Baden vorgestellt. Es handelt sich um ein von mir, einem erfahrungsheilkundlich arbeitenden Allgemeinmediziner, aus dem Vegatest-Verfahren von H.W. Schimmel entwickelten Verfahren der Komplementärmedizin. Die Methode wird mittlerweile von rund 700 speziell ausgebildeten Therapeuten in über 20 Ländern weltweit angewandt [1]. Bei den PSE-Therapeuten handelt es sich in der Mehrzahl um naturheilkundlich arbeitende Ärzte und Heilpraktiker, die in ihren Praxen chronisch Kranke betreuen.

Die PSE versteht sich dabei nicht als Rivale der universitär ausgerichteten «Schulmedizin», sondern als ihre Ergänzung. Die Erfahrung zeigt, dass die Schulmedizin notgedrungen Grenzen hat, was man an der grossen Zahl von ihr schwer oder nicht therapierbarer chronischer Krankheiten sehen kann. Ausserdem erleben wir in der Allgemeinmedizin, dass viele Patienten keine fassbare organische Krankheit haben, sondern psychosomatisch krank sind - ein objektiv schwer fassbarer Bereich, wo die Schulmedizin notgedrungen an ihre Grenzen stösst.

Wer beispielsweise ständig müde ist und sich unwohl fühlt, bei dem findet der Schulmediziner erfahrungsgemäss meist keine objektivierbare Ursache, während wir mit der PSE z.B. eine Energieblockade durch einen verdrängten seelischen Konflikt erkennen können, nach dessen Auflösung sich der Patient wieder wohl und energie-

\title{
20 Jahre Psychosomatische Energetik - ein Rückblick
}

Abb. 1.

Dr. Reinhard Voll.

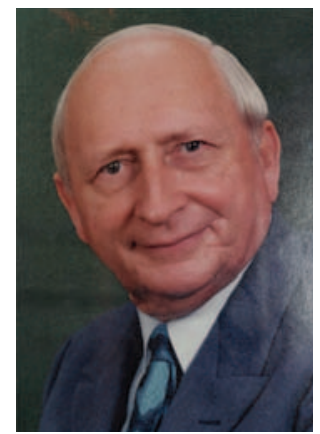

geladen fühlt. Oder wir finden bei solchen «Burnout»-Patienten mit der PSE einen energetisch gestörten Schlafplatz durch Elektrosmog und Erdstrahlen, nach dessen Sanierung der chronisch müde Patient wieder erholsam schläft und sich nach einigen Wochen wieder fit fühlt. Das Gleiche gilt in Abwandlung bei chronischen Krankheiten, wo wir den Krankheitsverlauf oft günstig beeinflussen oder sogar komplette Remissionen erleben können, etwa bei der chronischen Polyarthritis [2] oder der Colitis ulcerosa [3].

\section{Geschichte und Entwicklung der Methode}

Nach dem Zweiten Weltkrieg entwickelte der Plochinger Internist Dr. Reinhard Voll (Abb. 1) die Elektroakupunktur. Der Hauptfokus des Verfahrens lag - dem Zeitgeist entsprechend und ähnlich wie bei Dr. Reckeweg, dem Erfinder der Homotoxikologie auf energetischen Dissonanzen in Form von Stoffwechselgiften, die mit ausleitenden Medikamenten und Nosoden behandelt werden sollten. Der damalige beste Kenner der Nosoden,
Abb. 2.

Dr. Helmut W. Schimmel.

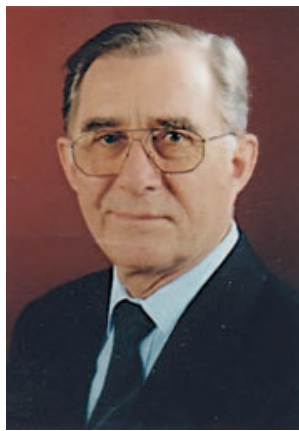

Dr. Helmut W. Schimmel (Abb. 2), mit dem ich jahrelang eng zusammengearbeitet habe, konnte energetische Dissonanzen durch Mischungen von homöopathischen Meridiankomplexen (Kern Pharma) behandeln, die mit den sieben Energiezentren des Yoga (Chakren) in Beziehung stehen.

Die Effekte dieses neuartigen Vorgehens waren beeindruckend, aber etwas Neues stiftete Verwirrung. Mit der Zeit kamen die Chakra-Störungen nämlich wieder, wobei die allermeisten Nosoden im Medikamententest nicht mehr ansprachen. Der Patient war entgiftet, aber immer noch krank. Die Harmonisierung der Chakren hatte offenbar die Nosoden-Signale gelöscht, aber etwas Unbekanntes hinter den Energiezentren hatte deren Reaktivierung und damit die erneuten Beschwerden der Patienten verursacht. Mit der Zeit wurde durch intensive Forschungsarbeit klar, dass hinter der wiederkehrenden Chakra-Störung seelische Konflikte als ursächlich angesehen werden mussten.

Erfahrungsgemäss sind die meisten Energieblockaden seelischer Natur und entstehen nach Auffassung der PSE durch lang zurückliegende seeli-

\section{KARGER}

(C) 2017 S. Karger GmbH, Freiburg

Fax +497614520714
Dr. med. Reimar Banis

Facharzt für Allgemeinmedizin

c/o IGPSE

Dörflistrasse 4, 6056 Kägiswil, Schweiz

drbanis@rubimed.com 
PSYCHOSOMATISCHE ENERGETIK EMOTIONALE KONFLIKTE UND ZUGEHÖRIGE ENERGIEZENTREN

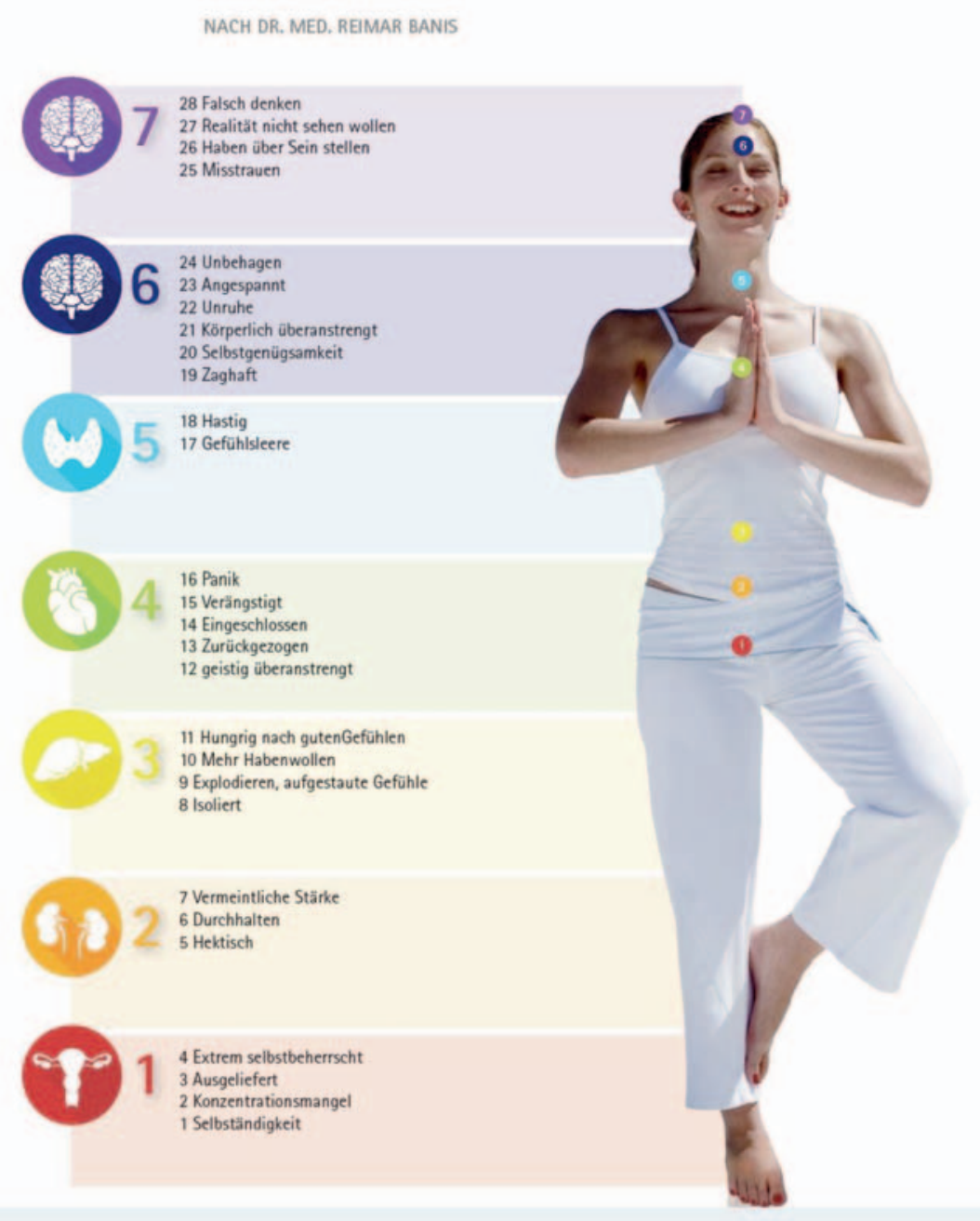

Abb. 3. Konfliktthemen und zugehörige Energiezentren.

sche Traumata. Diese schockartig wirkenden emotionalen Verletzungen werden in Energieblockaden gespeichert. Das ist damit vergleichbar, wie ein Organismus sich durch einen Masern-Hautausschlag von Viren befreit und diese Fremdkörper in die Peripherie des Organismus auslagert. Dort liegen die Energieblockaden für sehr lange Zeiträume, werden irgendwann passiv und sind energetisch einigermassen kompensiert. Seelisch gelangen sie synonym ins Unbewusste.

Mit Nosoden gehen die Konflikte teilweise in energetische Resonanz, aber das leider nur unvollständig, weshalb ich neue Komplexmittel entwi- mene wird der Konflikt mit der Zeit vollständig aufgelöst, was durch ein spezielles Testprocedere während der Behandlung, sofern nötig, jederzeit kontrolliert werden kann. Ist der Konflikt aufgelöst, steht dem Patient die darin gebundene Energie wieder zur Verfügung und er fühlt sich wieder vitaler. Gleichzeitig kommt es zu seelischen Selbstheilvorgängen hin $\mathrm{zu}$ mehr Autonomie.

Mit der effektiven Behandlung unbewusster Konflikte war die PSE entstanden. Wie aufgrund der Vorgeschichte zu erwarten, waren die meisten Nosoden damit therapeutisch unnötig geworden, indem der Patient durch die Auflösung der seelischen Konflikte zum Grossteil zusätzlich entgiftet wird.

\section{Erste Praxiserfahrungen mit der PSE und Festlegung fester Regeln}

Die ersten Erfahrungen mit der PSE in meiner Praxis waren unerwarteterweise unbefriedigend. Normalerweise erweisen sich gute testende Medikamente, die wie die Emotional- und Chakramittel der PSE im Energietest praktisch alle Energieebenen komplett ausgleichen, in der Anwendung als sehr effektiv. Unerwarteterweise zeigten jedoch Patienten, die nach einem halben Jahr zur Kontrolle zurückkamen, keine grosse Besserung, und oftmals wurde bei ihnen sogar ein neuer Konflikt getestet.

Erst mit der Zeit stellte sich heraus, worin die unbefriedigenden Therapieeffekte der Erprobungsphase ihre Ursache hatten und warum ständig neue Konflikte auftauchten. Ich hatte mehrere schwerwiegende Anfängerfehler bei der Anwendung der PSE begangen:

- Die meisten Konflikte benötigen zur vollständigen Auflösung 3-4 Monate oder länger, d.h., ich hatte sie viel zu kurz behandelt.

- Behandelt man Konflikte nur an, wie ich das unwissenderweise getan 


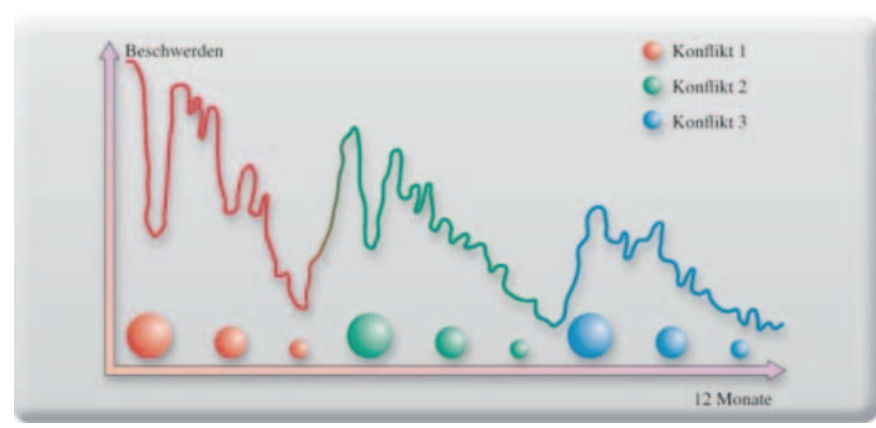

Abb. 4. Normaler Heilverlauf der PSE.

hatte, testet man bei der Kontrolle oft einen neuen Konflikt. Dadurch wird das psychoenergetische System immer konfuser, denn mehrere aktive, anbehandelte Konflikte wirken im Hintergrund weiter psychoenergetisch störend und potenzieren sich.

Als ich diese Fehler korrigiert hatte, stellte sich nach der Behandlung Hunderter Patienten heraus, dass man bei den meisten Patienten zur deutlichen Besserung oder Heilung nicht nur einen, sondern mehr Konflikte behandeln muss und dafür 12-16 Monate benötigt (Abb. 4). Während dieser Zeitspanne sollte der jeweilige Konflikt vollständig aufgelöst werden, d.h., es reicht meist keineswegs aus, nur einen einzigen Konflikt energetisch zu heilen, damit es dem Patienten deutlich und dauerhaft besser geht.

Hinzu kommt, dass die optimale PSE-Therapie eine konsequente Einnahmedisziplin des Patienten voraussetzt (zweimal täglich 12 Tropfen), denn nimmt er weniger ein, dauert es bis zur Konfliktheilung entsprechend länger. Nimmt er mehr ein, bringt das praktisch nichts. Zusätzlich wirksame oder andere effektive Formen der Konfliktheilung neben der PSE sind bis jetzt nicht gefunden worden, ausser der, dass Konflikte durch blosses Abwarten passiv werden können, aber dadurch bleiben sie trotzdem weiterhin eine potente Gefährdungsquelle. Ausserdem rauben sie ständig unterschwellig Lebensenergie und manipulieren auf negative Weise das relativ objektive Einschätzen einer Situation

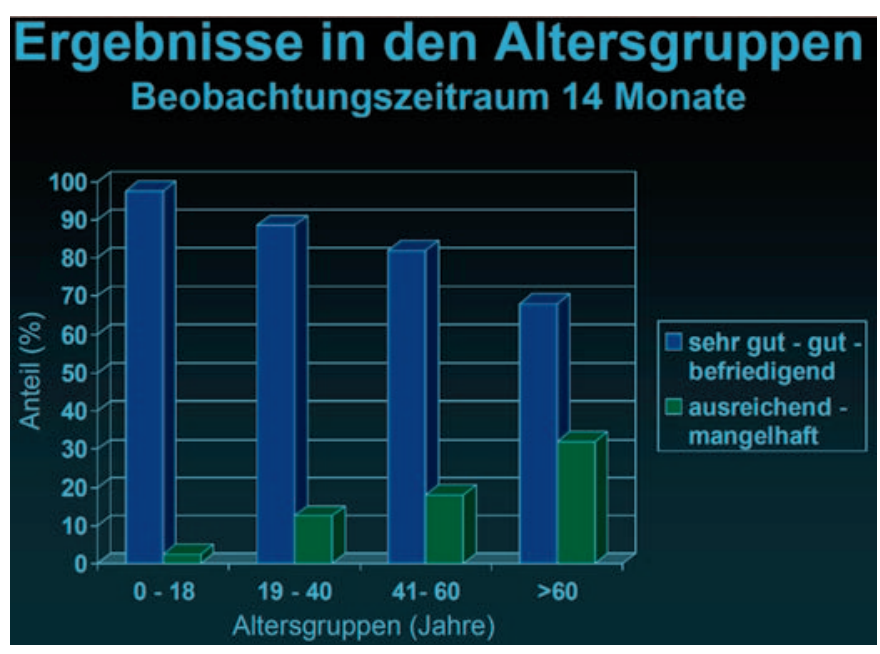

Abb. 5. Therapieerfolg mit der PSE sinkt in Abhängigkeit vom Alter (Studie «Langzeiterfolge mit der PSE» von Dr. med. B. Holschuh-Lorang, Vortrag am Expertentreffen Mai 2009 in Konstanz [4]).

und sabotieren damit indirekt das eigene Handeln.

$\mathrm{Zu}$ den festen Regeln der PSE gehört viel Disziplin vonseiten des Therapeuten. Er muss ein festes Testschema anwenden und ebenso ein genau $\mathrm{zu}$ befolgendes Therapieschema einhalten. Die Medikamente der PSE können nicht aufgeschwungen werden und sollten von Elektrosmog und anderen Störquellen wie zu grosser Hitze usw. ferngehalten werden. Detaillierte Informationen finden sich auf der Webseite der Internationalen Gesellschaft für Psychosomatische Energetik (IGPSE), einem als gemeinnützig anerkannten Fachverband mit Sitz in der Schweiz, sowie im offiziellen Lehrbuch der PSE [1].

\section{Jahre Erfahrung bei unterschiedlichsten Krankheitsbildern}

Mittlerweile überblicken wir 20 Jahre an Erfahrungen mit der PSE bei unterschiedlichsten Themen und bei allen Altersgruppen. (Eine von 2005 bis 2009 laufende multizentrische Praxisstudie mit 11 teilnehmenden Praxen und 1002 Patienten fasst die Behandlungsergebnisse zahlreicher Therapeuten zusammen, die sie mit der
PSE gewonnen haben. Die durchschnittliche Therapiedauer betrug 15 Monate mit insgesamt vier Konsultationen. Sehr gute, gute und befriedigende Therapieergebnisse wurden in $86,5 \%$ der Fälle erzielt. Wenn man berücksichtigt, dass in einer PSE-Praxis überwiegend Problempatienten behandelt werden, erscheint der erzielte Therapieerfolg umso beindruckender. Besonders gute Therapieerfolge sieht man bei Kindern und Jugendlichen sowie bei Patienten mit psychosomatisch geprägten Krankheitsbildern [4].) Grundsätzlich ist jede energetische Therapie umso wirksamer, je früher damit begonnen wird, wie Langzeitstudien der Allgemeinärztin Dr. Holschuh-Lorang gezeigt haben [5]. Die Therapieerfolge liegen bei Kindern bei über $90 \%$ und sinken danach auf unter 70\% bei über Sechzigjährigen (Abb. 5). Das entspricht der Erfahrung in der naturheilkundlichen Therapie, dass die Regenerationsfähigkeit altersabhängig ist. Konkret bedeutet das Ergebnis, dass man möglichst früh mit der Therapie beginnen sollte, wie auch Studien des Wiener Allgemeinarztes Dr. Richter an verhaltensgestörten Kindern eines Kinderheims gezeigt haben [6]. Vergleichbares hat der Internist Dr. Hemsing in einem anderen Kinderdorf unabhängig davon be- 
Abb. 6. Gerlinde Paukert.

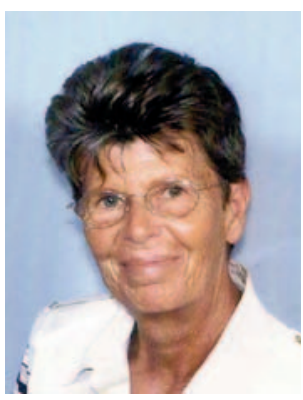

Abb. 7. Dr. Birgitt Holschuh-Lorang.

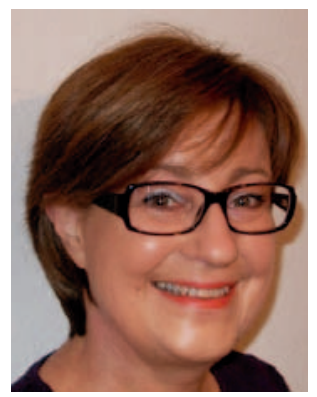

Die Domäne der PSE sind psychosomatische Krankheitsbilder wie Reizdarm, wobei oft die schulmedizinisch ignorierte Darmflora zusätzlich therapiert werden muss. Dazu zählen auch chronische Schmerzen etwa beim Weichteilrheuma, wo die PSE laut dem Rheumatologen Dr. Scharm eine hohe Erfolgsquote erreicht. Sein Fazit: «Beim Weichteilrheuma (Fibromyalgie) kann nach meiner mehrjährigen Praxiserfahrung die komplementärmedizinische Methode der PSE eine grosse Hilfe sein. In der Mehrzahl der Fälle kommt es zu einer deutlichen Besserung des Krankheitsbildes, wonach die Lebensqualität dauerhaft verbessert werden kann, was auch nach der Beendigung der PSE-Therapie anhält. Sehr häufig zeigt sich bei der PSETestung ein charakteristisches Muster in Form eines gestörten sechsten Energiezentrums (Hypothalamus, Vegetativum) mit dem Konflikt <Unbehagen>. Beides deckt sich mit dem Beschwerdebild der Fibromyalgie» [8].

Besonders herausgehoben wird von allen erfahrenen ärztlichen Kollegen, dass die einmal erzielten Therapieerfolge meist dauerhafter Natur sind, wie langjährige Nachbeobachtungen von Dr. Holschuh-Lorang (Abb. 7) bei ihren Patienten gezeigt haben (Praxisstudie mit 153 Patienten, die zwischen 2001 und 2009 mindestens 3 Jahre lang in einer allgemeinmedizinischen Praxis mit der PSE behandelt wurden. Frage: Wurde das Beschwerdebild tatsächlich dauerhaft beseitigt? $72 \%$ aller Fälle konnten mit sehr gut und gut, $12 \%$ mit befriedigend beurteilt werden. Nur 16\% aller Fälle erhielten eine ausreichende oder mangelhafte Be- wertung. Das seelische Gleichgewicht der meisten Patienten wurde wesentlich verbessert). Hinzu kommt ein seelischer Nachreifungsprozess hin $\mathrm{zu}$ mehr seelischer Autonomie, wie der Berliner Allgemeinarzt Dr. Müller immer wieder bei seinen Patienten beobachten konnte [9].

Betont werden sollte auch, dass die PSE die psychoenergetische Befindlichkeit von Menschen korrekt wiedergibt. Man kann dies daran erkennen, dass sich in Umfragen etwa ein Drittel einer nicht selektierten Bevölkerungsgruppe als krank, müde usw. bezeichnet, sich dagegen zwei Drittel als gesund mit einem guten Allgemeinbefinden beschreiben. Das deckt sich genau mit den Ergebnissen der PSE, während viele konkurrierende Naturheilverfahren entweder zu viele Menschen als krank und gestört oder im Gegenteil schulmedizinische Verfahren zu viele Menschen mit deutlichen Befindlichkeitsstörungen als scheinbar gesund bezeichnen. Die PSE entspricht dagegen der Realität solcher Umfragen - im praktischen Alltag ein sehr wichtiges Qualitätskriterium.

\section{PSE-Therapie bei Tieren}

Als wortlos arbeitende Form der Psychotherapie eignet sich die PSE besonders bei der Tierbehandlung. Bei verhaltensgestörten Haustieren haben die psychoenergetischen Störungen der Besitzer oft eine Verstärkungsfunktion, weshalb es ratsam erscheint, Tier und Herr in manchen Fällen gemeinsam zu behandeln. Tiere sprechen schnell und deutlich auf die Methode an, und nach den bisher gemachten Erfahrungen sind einmal aufgelöste Konflikte dauerhaft verschwunden. Mittels persönlicher Mitteilungen habe ich von einigen TierTherapeuten erfahren, dass sie speziell beim Leistungssport von Tieren gute Erfahrungen gemacht haben. cke von wenigen Metern bei Nikotinabusus, wo wir mit der PSE beeindruckende Erfolge gesehen haben. 


\section{PSE und Charaktertyp}

Ein besonders grosser Konflikt wird in der PSE als Zentralkonflikt bezeichnet, weil er eine zentrale Bedeutung für das Stoffwechselsystem des Patienten und auch für seinen Charakter hat. Das Chakra, dem der Zentralkonflikt zugeordnet wird, ist dabei entscheidend für das Temperament des Patienten:

- Sanguiniker $($ Hysteriker $)=$ Chakra 2 und 6 ,

- Phlegmatiker (zwanghafter Typ) Chakra 5,

- Choleriker (depressiver Typ) Chakra 3 oder

- Melancholiker (schizoider Typ) = Chakra 1 und 7.

Mit der Typbestimmung erhält der Therapeut ein wertvolles Hilfsmittel zur tiefenpsychologischen Beratung, weil man durch die PSE-Testung viele unbewusste Wesenszüge der Persönlichkeit daraus ableiten kann, die man sonst nur nach intimer Kenntnis einer Person erhält. Dank der Kenntnis des Temperaments kann dem Patient eine typgerechte Lebensberatung angeboten werden. Jeder Charakter hat bestimmte «Laster», die gemieden, und «Tugenden», die gefördert werden sollten. Am besten gelingt eine seelische Reifung durch die Abschmelzung des Zentralkonflikts, aber der Patient muss selbstverständlich auch selbst etwas mit dazu beitragen, damit es seelische Fortschritte gibt.

\section{Literatur}

1 Banis R: Lehrbuch Psychosomatische Energetik. Stuttgart, Haug, 2014.

2 Scharm M: Haben Polyarthritiker häufig eine bestimmte Persönlichkeit? Lesebuch der Psychosomatischen Energetik, Band 5. Books on demand, 2015.

3 Scharm M: Erfolgreiche Behandlung chronisch entzündlicher Darmerkrankungen mit der PSE. Vortrag bei der Medizinischen Woche Baden-Baden, 2016.
Die vier Charaktertypen haben sich in der täglichen Praxis als ausserordentlich hilfreiches Beratungsinstrument erwiesen - sei es, um sich über seine eigenen Schattenseiten klar zu werden oder um die Beziehung zu anderen Menschen zu analysieren. Auch in der Berufs-, Paar- und Kinderberatung hat sich dieses Konzept sehr bewährt. Verschiedene Hinweise sprechen ausserdem dafür, dass die Entstehung des Zentralkonfliktes auf frühere Inkarnation zurückgeführt werden kann (Näheres siehe «Durch Energieheilung zu neuem Leben»). Die PSE hat durch dieses Postulat eine viel grössere Tiefendimension bekommen, die weit in die Tiefenpsychologie und transpersonale Psychologie hineinreicht. Mit einiger Berechtigung kann man sogar von einer spirituellen Beratung sprechen, die dadurch möglich geworden ist (Näheres siehe «Spirituelle Energiemedizin»).

\section{Fazit}

Die PSE ist ein seit 20 Jahren bewährtes Verfahren der Komplementärmedizin, das sich als Standardverfahren zur Harmonisierung und Anhebung der Lebensenergie bewährt hat. Die als Prana, Ch'i, Aether usw. bezeichnete Lebensenergie scheint nicht nur geistiger, sondern vor allem energetischer Natur zu sein. Das sollte man begrifflich sauber trennen, denn

4 Banis R: Multizentrische Praxisstudie zur Psychosomatischen Energetik. Schweiz Z Ganzheitsmed 2010;5:269-272.

5 Holschuh-Lorang B: Wie stabil sind Heilerfolge? Langzeitstudienergebnisse unter Anwendung der Psychosomatischen Energetik. Vortrag am Expertentreffen im Mai 2009 in Konstanz.

6 Richter H: PSE-Therapie im Kinderdorf Pöttsching. Lesebuch der Psychosomatischen Energetik, Band 5. Books on demand, 2015. wer sich allgemein schlecht fühlt, dem hilft daher oft weniger ein Gespräch als eine Energiebehandlung. Wer nämlich viel Energie hat, fühlt sich kraftvoll, belastbar und gut gelaunt. Bei erkrankten und seelisch überlasteten Menschen findet sich dagegen meist zu wenig Energie. Die Energie wiederum wirkt als Taktgeber für jede Körperzelle, ist aber auch emotional anregend, hat also neben der seelischen auch eine wichtige körperliche Auswirkung - ein interessantes, wissenschaftlich noch wenig erforschtes Wechselspiel, das für unsere Lebensqualität von entscheidender Bedeutung zu sein scheint.

Seelische Konflikte scheinen unsichtbaren Computerviren vergleichbar das feine Zusammenspiel von Energie und Körper-Seele zu stören. Löst man die unbewussten Konflikte durch ähnliche (homöopathische) Schwingungsmuster über mehrere Monate auf, kann die Lebensenergie wieder frei fliessen und es kommt zur Aktivierung seelisch-körperlicher Selbstheilvorgänge. Bei allen gängigen allgemeinmedizinischen Krankheitsbildern sind dabei beeindruckende und lang anhaltende Verbesserungen des Befindens und erstaunlich oft Heilungen beobachtet worden.

Informationen zur PSE (Testsatz, Reba-Testgeräte, Emvita, Geovita): Rubimed AG, Grossmatt 3, 6052 Hergiswil, Schweiz, Tel. +41 041 / 6300888, Fax: -0887, www.rubimed.com.
7 Hemsing W: Adjuvante Homöopathie mit Tropfen bei verhaltensgestörten Jugendlichen. Lesebuch der Psychosomatischen Energetik, Band 3. Hochheim, Co'med, 2007.

8 Scharm M: Weichteilrheuma naturheilkundlich behandeln. Schweiz Z Ganzheitsmed 2016;28:89-91.

9 Müller A: Persönlichkeitsentwicklung mithilfe der Psychosomatischen Energetik. Schweiz Z Ganzheitsmed 2016;28:267-270. 